Address for Correspondence: Dr. Ai Peng, Department of Nephrology, Shanghai Tenth People's Hospital, Tongii University School of Medicine, Shanghai 200072, China.

Email: pengai@tongji.edu.cn

\begin{tabular}{|l|}
\hline Access this article online \\
\hline $\begin{array}{l}\text { Website: } \\
\text { www.intern-med.com }\end{array}$ \\
\hline DOI: \\
10.1515/jtim-2016-0031 \\
\hline Quick Response Code: \\
\hline \\
\\
\hline \\
回 \\
\hline
\end{tabular}

\title{
The Green Tea Polyphenol(-)- epigallocatechin-3-gallate and its beneficial roles in chronic kidney disease
}

\author{
Hui Bao, Ai Peng \\ Department of Nephrology, Shanghai Tenth People's Hospital, Tongji University School of Medicine, Shanghai, \\ China
}

\section{ABSTRACT}

Chronic kidney disease (CKD), a condition that affects around $10 \%$ of the population, has become a significant public health concern. Current therapeutic strategies to slow down the progression of CKD remain limited. Thus, it is urgent to develop new strategies to manage the patients with CKD. Work within the past decade has improved our understanding of the mechanisms contributing to CKD. In particular, oxidative stress as well as inflammation appears to play a pivotal role in CKD progression. (-)-Epigallocatechin-3-gallate (EGCG), the major catechin of green tea extract, is known as a powerful antioxidant and reactive oxygen species scavenger. Various studies have shown EGCG has a potential role in chronic kidney disease models. It is suggested that EGCG modulates cellular and molecular mechanisms via inflammation-related NF-KB and Nrf2 signaling pathway, as well as apoptosis-related ER stress pathway and mitochondrial pathway. Therefore, based on these studies, this review attempts to present a recent state of our knowledge and understanding of mechanisms of its role on the process of CKD, with the aim of providing some clues for the future optimization of EGCG in renal diseases.

Key words: chronic kidney disease, EGCG, green tea; inflammation; apoptosis

\section{INTRODUCTION}

Chronic kidney disease (CKD) is a longterm form of kidney disease, characterized by a progressive loss in renal function. The most common causes of CKD are diabetes mellitus in Western world and glomerulonephritis (GN) in Chin $a^{[1]}$. Others include hypertension, obstruction, druginduced CKD, and idiopathic nephropathy. Currently, therapeutic strategies to slow down the progression of CKD remain limited, like controlling risk factors, ACEI/ $\mathrm{ARB}$, and other conservative treatment $\mathrm{t}^{[2-4]}$. Thus, it is urgent to develop new therapeutic strategies to manage the patients with CKD.

Green tea, originally cultivated in China, is considered as one of the healthiest beverages in the world. (-)-Epigallocatechin-3-gallate (EGCG), the major catechin of green tea extract, is known as a powerful antioxidant and reactive oxygen species scavenger ${ }^{[5,6]}$ (chemical structure, Figure 1A). During the past decade, the health-promoting effects of EGCG have been intensively investigated. It is shown to inhibit leukocyte chemotaxis, quench free radicals, chelate transition metals, and interrupt lipid peroxidation chain reaction ${ }^{[7-9]}$. Kidney, exposed to harmful agents, is at high risk of oxidative stress, like reactive oxygen species (ROS) ${ }^{[10,11]}$. Hence oxidative stress and inflammation play a pivotal role in CKD progression $^{[12,13]}$. Evidence from numerous studies supports a potential role for EGCG in the renoprotective effect in CKD. It is demonstrated that EGCG decreased serum creatinine in rats with adenine-induced renal failure as a chronic kidney disease $\operatorname{model}^{[14]}$, and also improves glucose toxicity and renal injury in the animal model of diabetic nephropathy ${ }^{[15]}$.

Based on these considerations, EGCG, the natural compound, might be regarded as 


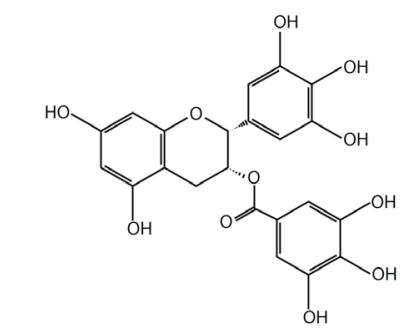

A. Structure of (-)-epigallocatechin-3-gallate (EGCG)

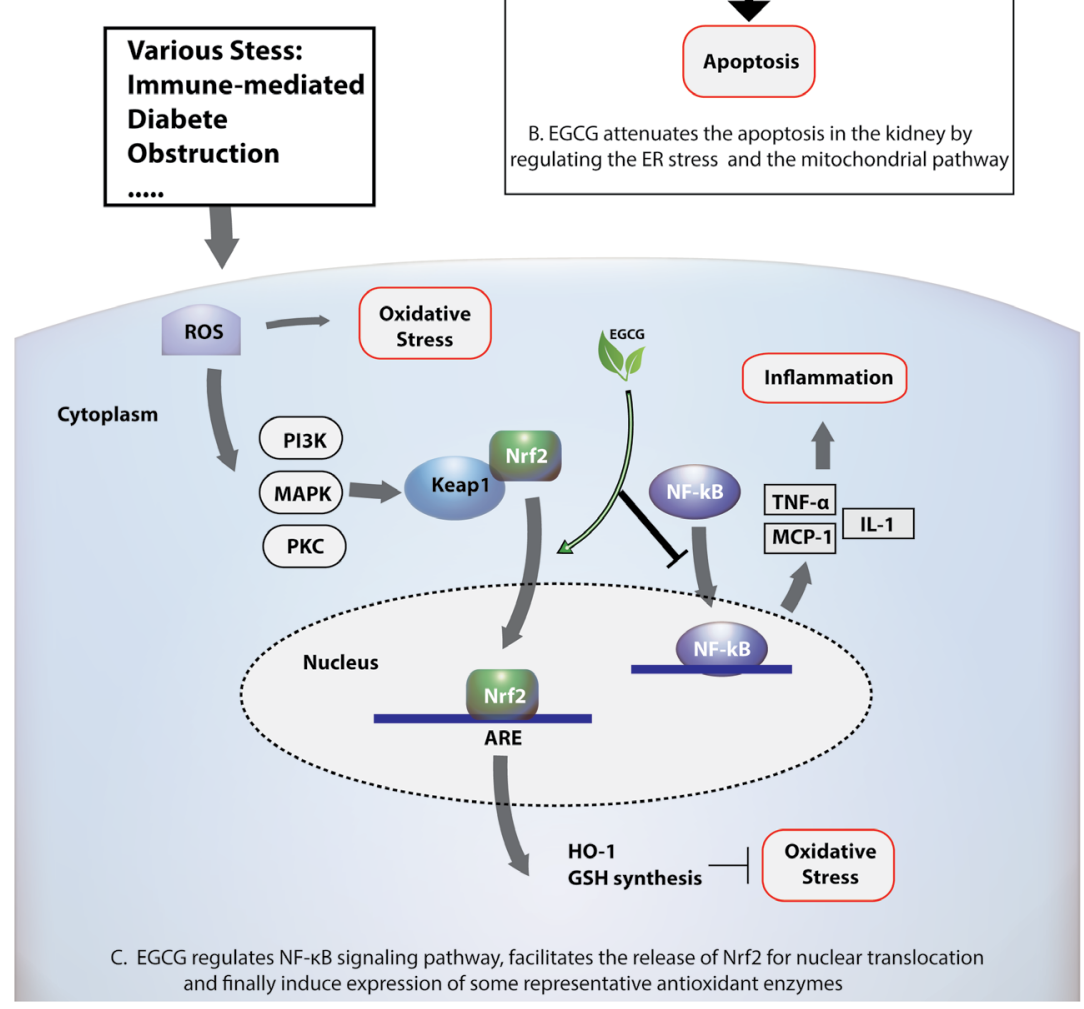

Figure 1 A: Structure of (-)-epigallocatechin-3-gallate (EGCG). B: Figure 1B: EGCG regulates NF-kB signaling pathway, facilitates the release of Nrf2 for nuclear translocation, and finally induces the expression of some representative antioxidant enzymes in renal tissue, like HO-1 and GSH synthesis. In addition, EGCG substantially suppressed the expression of inflammatory cytokines and hindered the generation of ROS. C: Figure 1C: EGCG attenuates the apoptosis in the kidney by regulating endoplasmic reticulum (ER) stress pathway and mitochondrial pathway. GRP78, glucose-regulated protein 78. ROS, reactive oxygen species; PI3K, phosphatidylinositol 3-kinase; MAPK, mitogen-activated protein kinase; PKC, proteinkinase C; Keap1, Kelch-like ECH-associated protein 1; Nrf2, nuclear factor erythroid 2-related factor 2; H0-1, heme oxygenase-1; TNF- $\alpha$, tumor necrosis factor- $\alpha$; MCP-1, monocyte chemotactic protein 1; GSH, glutathione.

a food component useful for the maintenance of renal health. In this review, we will present a recent state of our knowledge and understanding of mechanisms of its role on the process of $\mathrm{CKD}$, with the aim to provide some clues for the future optimization of EGCG in renal diseases.

\section{EGCG AND ITS POTENTIAL ROLE IN CKD}

Tea comes from the Camellia sinensis plant and is generally divided into categories based on different fermentation processes, like white tea, yellow tea, green tea, Oolong, and black tea, etc. During fermentation, catechins, the bioactive polyphenols in tea leaves, are converted into the aflavins and thearubiginsvia polyphenol oxidases.
Compared with other types of tea, green tea is unfermented and contains the highest concentration of catechins, in which EGCG accounts for as much as $50 \%$ of its total polyphenols and has been reported to possess antioxidative, antiinflammatory, and anticarcinogenic effects ${ }^{[6,16]}$. EGCG is estimated to be 25 times more potent than vitamin $\mathrm{E}$ and 100 times more potent than vitamin $\mathrm{C}^{[17]}$.

The kidney is essential in blood filtration, excretion of waste products, regulating the balance of electrolytes, along with maintaining homeostatic conditions, and thus at high risk of oxidative stress. ${ }^{[13]}$ As previously described, increasing studies indicated EGCG attenuated various renal injuries, specifically, immune-mediated GN, renal ischemia reperfusion, diabetic nephropathy, toxin-induced 
nephrotoxicity, and obstructive nephropathy. Various mechanisms have been proposed to explain the anti-CKD properties of EGCG due to their antioxidative properties and may provide hope for the successful treatment of the renal disorder.

\section{Immune-mediated renal injury}

GN, which accounts for $10-0 \%$ of the total incident cases of ESRD, is associated with increased morbidity and mortality. There are various types of GN that are classified based on the type of mechanisms and section of the glomeruli that are affected. The pathogenesis involved in GN can be considered reverently immune mediated, including both cellular (e.g., neutrophils, monocytes) and humoral (e.g., complement) mediated immunity. Among them, antiglomerular basement membrane glomerulonephritis (anti-GBM-GN) is one of the most severe forms of immune-mediated GN, which is directed against an antigen intrinsic to the alpha-3 chain of type IV collagen in the GBM, thereby resulting in rapidly progressive GN, which is typically associated with crescent formation ${ }^{[18,19]}$. The contemporary treatment based upon the use of corticosteroids and/or immunosuppressive drugs has given excellent results in improving the survival of both the patient and the kidney; however, this treatment is accompanied by many side effects. Thus, the rationale for an etiological treatment is still lacking.

EGCG are potent anti-inflammatory and antioxidant agents. Recently, it has attracted considerable attention because of its potential role in the pathogenesis of immune-mediated renal injury by targeting multiple signaling and inflammatory pathways. A first study was performed on a murine model of anti-GBM-GN to test whether the anti-inflammatory and antioxidant properties of EGCG favorably affect the course of immune-mediated GN. Pretreatment with EGCG leads to an unequivocal improvement in histological findings and a significant reduction in proteinuria and serum creatinine ${ }^{[20]}$. The detailed mechanism is likely to be involved in the decreased oxidative stress and inflammation due to the EGCG treatment. Lower levels of oxidative stress markers MDA and $\mathrm{H}_{2} \mathrm{O}_{2}$ was observed in kidney and urine. Also there was mild focus of NF- $x \mathrm{~B}$ and its downstream gene expression by EGCG ${ }^{[21,22]}$. Additionally, ERK1/2 activation, phosphatidylinositol 3-kinase/Akt, and nuclear factor erythroid 2-related factor 2 (Nrf2) signaling pathway is also involved in $\mathrm{EGCG}^{[23]}$. The latest research demonstrated improvements in renal function and histology and found to be accompanied by the restoration of $\mathrm{Nrf} 2$ signaling on experimental crescentic GN induced in 129/svJ mice, as shown by increased nuclear translocation of Nrf2, reduced p-Akt, p-JNK, p-ERK1/2, and p-P38 expressing as well as restoration of PPAR $\gamma$ and SIRT1 levels ${ }^{[24]}$. Furthermore, Tsai et al. found that EGCG has prophylactic effects on lupus nephritis in these mice that are highly associated with its effects of enhancing the Nrf2 antioxidant signaling pathway, decreasing renal NLRP3 inflammasome activation, and increasing systemic Treg cell activity ${ }^{[22,25]}$. Also, Peairs et al. showed that EGCG inhibits the immune-stimulated PI3K/Akt/mammalian target of rapamycin (mTOR) pathway and blocked LPS/ IFN- $\gamma$-induced inflammatory mediator production (iNOS expression, supernatant NO, and interleukin-6) in MRL/ lpr mouse mesangial cells ${ }^{[26]}$. Thus, these observations demonstrate the potential utility of EGCG as a therapeutic agent for regulating inflammation and controlling GN or other autoimmune disease.

\section{Diabetic nephropathy}

Diabetic nephropathy is one of the most serious complications in diabetes mellitus and has been the most common cause of ESRD ${ }^{[2]}$. Various pathways are activated within the diabetic kidney, such as increasing oxidative stress, polyol formation, and AGE accumulation, further stimulating intracellular secondary messengers such as proteinkinase $\mathrm{C}$, mitogen-activated protein kinase, and $\mathrm{NF}-x \mathrm{~B}$, eventually resulting in increasing proteinuria, glomerular sclerosis, and tubulointerstitial fibrosis ${ }^{[28,29]}$. Therefore, therapeutic strategies targeting the management and prevention of diabetic nephropathy include agents for controlling hyperglycemia, glucose-derived oxidative stress, and renal damage ${ }^{[30]}$.

Due to the dominant role of antioxidant and antiglycation properties, EGCG are increasingly being investigated in diabetic nephropathy ${ }^{[31,32]}$. In a study performed on streptozotocin-induced diabetic rats fed with diet enriched with EGCG for 50 days, it has been shown that EGCG reduced the level of mRNAs for glyconeogenesis enzymes, suppressed hyperglycemia, proteinuria, and lipid peroxidation in diabetic nephropathy ${ }^{[33]}$. In vivo studies from Chen $e$ al. showed that oral administration of a 1:1 mixture of EGCG and Amla extract (AE) for 3 months significantly improved antioxidant defense as well as diabetic and atherogenic indices in uremic patients with diabetes, without significant changes in hepatic or renal function $^{[34]}$. One hypothesis to explain these properties is EGCG reduced renal AGE accumulation and its related protein expression in the kidney cortex, which might contribute to alleviating renal damage. Moreover, Cai et al. indicated that carbonyl stress mediated the formation of ubiquitinated, modified protein aggregation is essential in the early development of diabetic nephropathy, while methylated derivative EGCG, which is more stable and efficient than EGCG, could decrease the accumulation of p62, the antiparallel b-sheet/aggregatedstrands, and increase the a-helix content in a diabetic kidney ${ }^{[35]}$. Another in vitro study conducted on AGE-treated human kidney 
cells demonstrated that EGCG increased superoxide dismutase levels, decreased inflammation and apoptosis through inhibition of RAGE up-regulation, and NF- $x \mathrm{~B}$ pathway activation ${ }^{[36]}$. These studies suggest that dietary supplementation with EGCG could potentially contribute to nutritional strategies and is a safe and effective treatment for the prevention and treatment of $\mathrm{DN}$.

\section{Other related nephropathy}

Obstructive nephropathy refers to the presence of structural or functional changes in the urinary tract that impede the normal flow of urine. It has been the most common cause of ESRD in children. Several groups have reported a reduction of serum creatinine on animal models of obstructive nephropathy by EGCG treatment ${ }^{[37]}$. To explain the mechanism of action, it has been proposed that EGCG regulates NF- $x \mathrm{~B}$ activation and induces $\mathrm{Nrf2}$ nuclear translocation ${ }^{[13,38,39]}$. In UUO C $57 \mathrm{BL} / 6$ mice models, it has been found that treatment with $50 \mathrm{mg} /$ $\mathrm{kg}$ EGCG(I.P.) for 14 days inhibits NF- $\varkappa \mathrm{B}$ activation and favored a significant increase in the phosphorylated $\mathrm{I} x \mathrm{~B}$ protein. The phenomenon of Nrf2 nuclear translocation was also observed in a study conducted on a rat model of obstructive nephropathy. EGCG treatment suppressed oxidative stress and acute renal damage in a dose-dependent manner due to induced expression of glutathione S-transferase, glutathione peroxidase, $\gamma$-GCS, and heme oxygenase-1(HO-1), which are involved in the elimination or inactivation of ROS and oxidative stress ${ }^{[38]}$. These results indicate that EGCG is able to block NF- $x \mathrm{~B}$ signaling pathway, facilitate the release of $\mathrm{Nrf} 2$ for nuclear translocation, and finally induce expression of some representative antioxidant enzymes in renal tissue.

Cisplatin (CP) is a commonly used anticancer drug, but its notable side effect of nephrotoxicity limits its use in clinic $^{[40]}$. As previously described, cisplatin-induced nephrotoxicity is associated with increased oxidative stress, inflammatory cytokines, and finally result in apoptosis. Several studies have shown that EGCG attenuated the CP-induced apoptosis in animal models and may represent new promising adjunct candidate for cisplatin ${ }^{[41-44]}$. The details of EGCG regulate the $\mathrm{CP}$-induced apoptosis is complex and involves multiple mechanisms. First, the imbalance in ER homeostasis, as multifunctional organelle, caused disturbance of calcium homeostasis, glucose deprivation, increasing of ROS formation and oxidative stress induction ${ }^{[45]}$. Chen et al. showed that EGCG decreased p-ERK, GRP78, caspase-12 of kidney, as ER stress-related markers via inhibition of ER stress-induced apoptosis ${ }^{[4]}$. Second, a recent study has examined the effect of protection again cisplatin induced renal injury by EGCG through mitochondrial protection by improving mitochondrial electron chain complexes, mitochondrial antioxidant function in enzymes MnSOD and GPX, and improving cisplatin-induced mitochondrial oxidative/nitrative damage and anti-inflammatory effect ${ }^{[37}$. In a mouse model of cisplatin nephrotoxicity, EGCG ameliorate cisplatin induced apoptosis by modulating death of receptor Fas, Bax, and Bcl-2 $2^{[47]}$. In addition to that, EGCG attenuated cisplatin-induced apoptotic cell death and mitochondrial ROS generation in human kidney tubular cell line HK-2 ${ }^{[37]}$. These findings suggest that EGCG can ameliorate $\mathrm{CP}$-induced apoptosis in the kidney by regulating the ER stress pathway and the mitochondrial pathway.

\section{CONCLUSIONS AND PERSPECTIVES}

On the basis of these studies, the beneficial properties of EGCG on CKD have been established and may have a high potential use in the prevention and treatment of CKD. The detailed mechanisms, although not fully understood, likely involve signaling pathways that are shared with inflammation-related $\mathrm{NF}-x \mathrm{~B}$ and $\mathrm{Nrf} 2$ signaling pathways (Figure 1C), as well as apoptosis-related the ER stress pathway and the mitochondrial pathway (Figure 1B). Nevertheless, to prove the effectiveness and safety use of EGCG, clinical studies investigating the effects of EGCG on CKD would be necessary.

\section{Source of Foundation}

This work was partially sponsored by the Natural Science Foundation of China (No. 81270136, No. 81500508, No. 81671897), Shanghai Pujiang Program (No. 15PJ1406800).

\section{Conflict of Interest}

None declared.

\section{REFERENCES}

1. Coresh J, Astor BC, Greene T, Eknoyan G, Levey AS. Prevalence of chronic kidney disease and decreased kidney function in the adult US population: Third National Health and Nutrition Examination Survey. Am J Kidney Dis 2003; 41:1-12.

2. Sharma P, Blackburn RC, Parke CL, McCullough K, Marks A, Black C. Angiotensin-converting enzyme inhibitors and angiotensin receptor blockers for adults with early (stage 1 to 3 ) non-diabetic chronic kidney disease. Cochrane DB Syst Rev 2011:CD007751.

3. Fraser SD, Taal MW. Multimorbidity in people with chronic kidney disease: implications for outcomes and treatment. Curr Opin Nephrol Hypertens 2016.

4. Meguid El Nahas A, Bello AK: Chronic kidney disease: the global challenge. Lancet 2005, 365:331-40.

5. Yang CS, Chen L, Lee MJ, Balentine D, Kuo MC, Schantz SP. Blood and urine levels of tea catechins after ingestion of different amounts of green tea by human volunteers. Cancer Epidemiol Biomarkers Prev 1998; 7:351-4.

6. Higdon JV, Frei B. Tea catechins and polyphenols: health effects, metabolism, and antioxidant functions. Crit Rev Food Sci Nutr 2003; 43:89-143.

7. Takano K, Nakaima K, Nitta M, Shibata F, Nakagawa H. Inhibitory effect of (-)-epigallocatechin 3-gallate, a polyphenol of green tea, on neutrophil 
chemotaxis in vitro and in vivo. J Agr Food Chem 2004; 52:4571-6.

8. Katiyar SK, Matsui MS, Elmets CA, Mukhtar H. Polyphenolic antioxidant (-)-epigallocatechin-3-gallate from green tea reduces UVB-induced inflammatory responses and infiltration of leukocytes in human skin. Photochem Photobiol 1999; 69:148-53.

9. Lambert JD, Elias RJ. The antioxidant and pro-oxidant activities of green tea polyphenols: a role in cancer prevention. Arch Biochem Biophys 2010; 501:65-72.

10. Pedraza-Chaverri J, Sanchez-Lozada LG, Osorio-Alonso H, Tapia E, Scholze A. New Pathogenic Concepts and Therapeutic Approaches to Oxidative Stress in Chronic Kidney Disease. Oxid Med Cell Longev 2016; 2016:6043601.

11. Vilaysane A, Chun J, Seamone ME, Wang W, Chin R, Hirota S, et al. The NLRP3 inflammasome promotes renal inflammation and contributes to CKD. J Am Soc Nephrol 2010; 21:1732-44.

12. Yoon JW, Pahl MV, Vaziri ND. Spontaneous leukocyte activation and oxygen-free radical generation in end-stage renal disease. Kidney Int 2007; 71:167-72.

13. Kim HJ, Vaziri ND. Contribution of impaired Nrf2-Keap1 pathway to oxidative stress and inflammation in chronic renal failure. Am J Physiol Renal Physiol 2010; 298:F662-71.

14. Nakagawa T, Yokozawa T, Sano M, Takeuchi S, Kim M, Minamoto S. Activity of (-)-epigallocatechin 3-O-gallate against oxidative stress in rats with adenine-induced renal failure. J Agr Food Chem 2004; 52:2103-7.

15. Yoon SP, Maeng YH, Hong R, Lee BR, Kim CG, Kim HL, et al. Protective effects of epigallocatechin gallate (EGCG) on streptozotocin-induced diabetic nephropathy in mice. Acta Histochemica 2014; 116:1210-5.

16. Dufresne CJ, Farnworth ER: A review of latest research findings on the health promotion properties of tea. The Journal of nutritional biochemistry 2001, 12:404-21.

17. Clark J, You M. Chemoprevention of lung cancer by tea. Mol Nutr Food Res 2006; 50:144-51.

18. Okamoto H. Molecular architecture of the Goodpasture autoantigen. $\mathrm{N}$ Engl J Med 2010; 363:1770; author reply 1.

19. Little MA, Pusey CD. Rapidly progressive glomerulonephritis: current and evolving treatment strategies. J Nephrol 2004; 17 Suppl 8: S10-9.

20. Peng A, Ye T, Rakheja D, Tu Y, Wang T, Du Y, et al. The green tea polyphenol (-)-epigallocatechin-3-gallate ameliorates experimental immunemediated glomerulonephritis. Kidney Int 2011; 80:601-11.

21. Tomita N, Morishita R, Lan HY, Yamamoto K, Hashizume M, Notake $\mathrm{M}$, et al. In vivo administration of a nuclear transcription factor-kappaB decoy suppresses experimental crescentic glomerulonephritis. J Am Soc Nephrol 2000; 11:1244-52.

22. Tsai PY, Ka SM, Chang JM, Chen HC, Shui HA, Li CY, et al. Epigallocatechin-3-gallate prevents lupus nephritis development in mice via enhancing the Nrf2 antioxidant pathway and inhibiting NLRP3 inflammasome activation. Free Radic Biol Med 2011; 51:744-54.

23. Wu CC, Hsu MC, Hsieh CW, Lin JB, Lai PH, Wung BS. Upregulation of heme oxygenase-1 by Epigallocatechin-3-gallate via the phosphatidylinositol 3-kinase/Akt and ERK pathways. Life Sci 2006; 78:2889-97.

24. Ye T, Zhen J, Du Y, Zhou JK, Peng A, Vaziri ND, et al. Green tea polyphenol (-)-epigallocatechin-3-gallate restores Nrf2 activity and ameliorates crescentic glomerulonephritis. PloS one 2015; 10:e0119543.

25. Liu X, Bao C, Hu D. Elevated interleukin-18 and skewed Th1:Th2 immune response in lupus nephritis. Rheumatol Int 2012; 32:223-9.

26. Peairs A, Dai R, Gan L, Shimp S, Rylander MN, Li L, et al. Epigallocatechin-3-gallate (EGCG) attenuates inflammation in MRL/lpr mouse mesangial cells. Cell Mol Immunol 2010; 7:123-32.

27. John S. Complication in diabetic nephropathy. Diabetes Metab Syndr 2016.

28. Bhatti AB, Usman M. Drug Targets for Oxidative Podocyte Injury in Diabetic Nephropathy. Cureus 2015; 7:e393.

29. Kawanami D, Matoba K, Utsunomiya K. Signaling pathways in diabetic nephropathy. Histol Histopathol 2016; 31:1059-67.
30. Gnudi L, Coward RJ, Long DA. Diabetic Nephropathy: Perspective on Novel Molecular Mechanisms. Trends Endocrinol Metab 2016.

31. Orsolic N, Sirovina D, Gajski G, Garaj-Vrhovac V, Jazvinscak Jembrek M, Kosalec I. Assessment of DNA damage and lipid peroxidation in diabetic mice: effects of propolis and epigallocatechin gallate (EGCG). Mutat Res 2013; 757:36-44.

32. Wolfram S, Raederstorff D, Preller M, Wang Y, Teixeira SR, Riegger C, et al. Epigallocatechin gallate supplementation alleviates diabetes in rodents. J Nutr 2006; 136:2512-8.

33. Yamabe N, Yokozawa T, Oya T, Kim M. Therapeutic potential of (-)-epigallocatechin 3-O-gallate on renal damage in diabetic nephropathy model rats. J Pharmacol Exp Ther 2006; 319:228-36.

34. Chen TS, Liou SY, Wu HC, Tsai FJ, Tsai CH, Huang CY, et al. Efficacy of epigallocatechin-3-gallate and Amla (Emblica officinalis) extract for the treatment of diabetic-uremic patients. J Med Food 2011; 14:718-23.

35. Cai S, Zhong Y, Li Y, Huang J, Zhang J, Luo G, et al. Blockade of the formation of insoluble ubiquitinated protein aggregates by EGCG3"Me in the alloxan-induced diabetic kidney. PloS one 2013; 8:e75687.

36. Liang YJ, Jian JH, Liu YC, Juang SJ, Shyu KG, Lai LP, et al. Advanced glycation end products-induced apoptosis attenuated by PPARdelta activation and epigallocatechin gallate through NF-kappaB pathway in human embryonic kidney cells and human mesangial cells. Diabetes Metab Res Rev 2010; 26:406-16.

37. Pan H, Chen J, Shen K, Wang X, Wang P, Fu G, et al. Mitochondrial modulation by Epigallocatechin 3-Gallate ameliorates cisplatin induced renal injury through decreasing oxidative/nitrative stress, inflammation and NF-kB in mice. PloS one 2015; 10:e0124775.

38. Zhou P, Yu JF, Zhao CG, Sui FX, Teng X, Wu YB. Therapeutic potential of EGCG on acute renal damage in a rat model of obstructive nephropathy. Mol Med Rep 2013; 7:1096-102.

39. Truong LD, Choi YJ, Tsao CC, Ayala G, Sheikh-Hamad D, Nassar G, et al. Renal cell apoptosis in chronic obstructive uropathy: the roles of caspases. Kidney Int 2001; 60:924-34.

40. Sastry J, Kellie SJ. Severe neurotoxicity, ototoxicity and nephrotoxicity following high-dose cisplatin and amifostine. Pediatr Hemat Oncol 2005; 22:441-5.

41. El-Mowafy AM, Al-Gayyar MM, Salem HA, El-Mesery ME, Darweish MM. Novel chemotherapeutic and renal protective effects for the green tea (EGCG): role of oxidative stress and inflammatory-cytokine signaling. Phytomedicine. Int J Phytother Phytopharmacol 2010; 17:1067-75.

42. Sahin K, Tuzcu M, Gencoglu H, Dogukan A, Timurkan M, Sahin N, et al. Epigallocatechin-3-gallate activates $\mathrm{Nrf2} / \mathrm{HO}-1$ signaling pathway in cisplatin-induced nephrotoxicity in rats. Life Sci 2010; 87:240-5.

43. Fatima S, Al-Mohaimeed N, Al-Shaikh Y, Tyagi P, Banu N, Hasan S, et al. Combined treatment of epigallocatechin gallate and Coenzyme Q10 attenuates cisplatin-induced nephrotoxicity via suppression of oxidative/ nitrosative stress, inflammation and cellular damage. Food Chem Toxicol 2016; 94:213-20.

44. Khan SA, Priyamvada S, Khan W, Khan S, Farooq N, Yusufi AN. Studies on the protective effect of green tea against cisplatin induced nephrotoxicity. Pharmacol Res 2009; 60:382-91.

45. Liu H, Baliga R: Endoplasmic reticulum stress-associated caspase 12 mediates cisplatin-induced LLC-PK1 cell apoptosis. J Am Soc Nephrol 2005; 16:1985-92.

46. Chen B, Liu G, Zou P, Li X, Hao Q, Jiang B, et al. Epigallocatechin-3-gallate protects against cisplatin-induced nephrotoxicity by inhibiting endoplasmic reticulum stress-induced apoptosis. Exp Biol Med 2015; 240:1513-9.

47. Zou P, Song J, Jiang B, Pei F, Chen B, Yang X, et al. Epigallocatechin-3-gallate protects against cisplatin nephrotoxicity by inhibiting the apoptosis in mouse. Int J Clin Exp Pathol 2014; 7:4607-16.

How to cite this article: Bao H, Peng A. The Green Tea Polyphenol (-)-epigallocatechin-3-gallate and its beneficial roles in chronic kidney disease. J TransI Intern Med 2016; 4: 99-103. 